\title{
Factorial design and post-antibiotic sub-MIC effects of linezolid combined with fosfomycin against vancomycin-resistant enterococci
}

\author{
Yisong Yan ${ }^{1,2}$, Guang Yang ${ }^{1,3}$, Yaowen $\mathrm{Li}^{1}$, Jun $\mathrm{Mao}^{1}$, Shuaishuai Wang ${ }^{1}$, Na Zhang ${ }^{1}$, Huiping Liu ${ }^{1}$, \\ Xiaohui Huang ${ }^{1}$
}

${ }^{1}$ Department of Basic and Clinical Pharmacology, School of Pharmacy, Anhui Medical University, Hefei, China; ${ }^{2}$ Department of Pharmacy, Anhui College of Traditional Chinese Medicine, Wuhu, China; ${ }^{3}$ Department of Pharmacy, The Third People's Hospital of Tongling, Tongling, China

Contributions: (I) Conception and design: Y Yan, X Huang; (II) Administrative support: X Huang; (III) Provision of study materials or patients: G Yang; (IV) Collection and assembly of data: Y Li, N Zhang, H Liu; (V) Data analysis and interpretation: J Mao, S Wang; (VI) Manuscript writing: All authors; (VII) Final approval of manuscript: All authors.

Correspondence to: Xiaohui Huang. Department of Basic and Clinical Pharmacology, School of Pharmacy, Anhui Medical University, Hefei 230032, China. Email: math2088@163.com.

Background: Antimicrobial drug resistance, including vancomycin-resistant enterococci (VRE), has long been an inescapable clinical problem. If vancomycin loose its therapeutic relevance, a regimen of linezolid combined with fosfomycin may provide an alternative option.

Methods: In this study, the in vitro antimicrobial effect of linezolid combined with fosfomycin on several different types of VRE was investigated using a checkerboard method and time-kill assays. Based on the results of the $24 \mathrm{~h}$ time-kill assays, a $2^{2}$ factorial design was then adopted. Finally, the post-antibiotic effect (PAE), post-antibiotic sub-minimum inhibitory concentration effect (PASME), and single sub-minimum inhibitory concentration effect (SME) of a combination of the two drugs on three selected strains was examined.

Results: The checkerboard method and factorial design analysis showed that linezolid combined with fosfomycin not only had synergistic and additive effects but also had an interactive effect on VREs. The time-kill assays showed that $1 \times$ minimum inhibitory concentration (MIC) of linezolid combined with $1 \times$ MIC or 1/4× MIC of fosfomycin had no statistically significant difference in the bactericidal effect against VRE at $24 \mathrm{~h}(\mathrm{P}>0.05)$. The combination of the two drugs did not significantly extend the PAE or the SME; however, in relation to $1 / 4 \times$ MIC, the combination of the two drugs significantly prolonged the duration of the PASME compared to that of a single drug on VREs $(\mathrm{P}<0.05$; with values of $1.97 \pm 0.01,4.32 \pm 0.18$, and $1.74 \pm 0.13$ h, respectively).

Conclusions: Our results showed that when linezolid is selected for the treatment of VRE infections, subinhibitory concentrations of fosfomycin can be administered at multiple intervals to improve the therapeutic effect.

Keywords: Vancomycin-resistant enterococci (VRE); linezolid; fosfomycin; interaction; post-antibiotic Sub-MIC effect

Submitted Aug 11, 2021. Accepted for publication Sep 27, 2021.

doi: 10.21037/atm-21-4595

View this article at: https://dx.doi.org/10.21037/atm-21-4595 


\section{Introduction}

In 1988, the first clinical isolates of vancomycin-resistant enterococci (VRE) were reported in Europe $(1,2)$. These then spread rapidly to the United States, Asia, and South America (3). The main mechanism of resistance to vancomycin is due to the presence of enzymes that produce low-affinity binding precursors in which carboxy-terminal $\mathrm{D}$-alanine residues are replaced by $\mathrm{D}$-lactate or $\mathrm{D}$-serine, leading to a reduction in binding affinity (4). Depending on the phenotype and genotype, VRE can be classified into 6 types. Of which, 5 types (VanA, VanB, VanD, VanE, and VanG) correspond to acquired resistance, and the remaining type (VanC) is an intrinsic property of Enterococcus (E.) gallinarum and E. casseliflavus. Since the classification of glycopeptide resistance is based on the primary sequence of the structural genes for the anti-ligase, rather than the level of resistance to the glycopeptide, different types of VRE have high or low minimum inhibitory concentration (MIC) to vancomycin (5).

Among the many infections caused by enterococci, the rate and severity of VRE is very high (6). Enterococci can cause bloodstream infections associated with central venous catheters, urinary tract infections associated with urethral catheters, and skin and soft tissue infections (7). Concomitant with reports of drug resistance, current antimicrobial drugs for the treatment of VRE infections are limited, but include monotherapy regimens, such as tigecycline, linezolid, daptomycin, platensimycin, and fosfomycin (8), or drug combinations, which are often recommended for the treatment of severe and highly drugresistant infections (9). In clinical practice, linezolid, a special grade use antimicrobial drug, is generally used as a single agent in the treatment of infections; its combination with the relatively inexpensive and more readily available fosfomycin for the treatment of serious infections has not been adopted clinically. The feasibility of the combination of the 2-drug is still in the basic research stage, and our study was conducted from this point of view.

Linezolid is an oxazolidinone approved for the treatment of community- and hospital-acquired pneumonia and VRE infections (10). It binds to the $23 \mathrm{~S}$ ribosomal ribonucleic acid (RNA) of the bacterial 50S subunit to prevent the formation of an initiation complex in protein synthesis, resulting in antibacterial activity against enterococci (11). As single agents continue to be used in clinical settings, reports of linezolid-resistant enterococcal infections and their hospital-transmitted history have followed (12). Risk factors for the development of resistance include repeated and long-term treatment (12). Fosfomycin also has an antibacterial effect against resistant gram-positive bacteria, such as methicillin-resistant Staphylococcus aureus and VRE (13). Due to its uncommon cross-resistance, fosfomycin is often used in combination with other drugs for severe infections to avoid the selection of resistant mutants (13). Qi et al. (14) suggested linezolid in combination with fosfomycin as a potential treatment for VRE infection. In a study of the synergistic combination of linezolid and fosfomycin, Jiang et al. (15) showed that the combination effectively inhibits the selection of enterococcal-resistant mutants by closing each other's mutant selection window. Given the limited treatment options available, new combination therapies of the two above-mentioned drugs represent a potential treatment for VRE.

The post-antibiotic effect (PAE) is a pharmacodynamic parameter that should be considered when selecting an antimicrobial dosing regimen (16). PAE is defined as the length of time that bacterial growth is inhibited after a brief exposure to an antimicrobial drug (16). MIC is defined as the lowest concentration of the drug that completely inhibits microbial growth. The sub-MIC is the concentration of the drug below the MIC. The effect of sub-MICs on bacterial growth during the PAE is defined as the post-antibiotic sub-MIC effect (PASME). The PASME encompasses the time for the PAE plus the additional time that sub-MIC concentrations inhibit growth (17). Conversely, the single sub-MIC effect (SME) measures the direct effect of subinhibition levels on bacteria not previously exposed to antimicrobial drugs. In clinical practice, there are often subMIC concentrations between intermittent administration, and it may be that PASME reflects drug metabolism in vivo more than PAE (18). Factorial design is a simple way of comparing the main effects of multiple independent variables and exploring possible interaction effects, which allows investigators to efficiently examine multiple independent variables (also known as factors) and examine both main and interaction effects (19).

Although studies of linezolid combined with fosfomycin against VREs have been reported, the optimization of the 2-drug combination in terms of dose and dosing interval has not been reported through studies related to the PAE, PASME, and SME. In this study, several different types of 
VRE were selected and their susceptibility to vancomycin was determined. The in vitro antibacterial effect of linezolid combined with fosfomycin on some strains was examined using broth micro-checkerboard testing and time-kill assays, and a factorial design was adopted based on the results of the time-kill assays to analyze whether there was an interaction between the two drugs (20). The PAE, PASME, and SME of the drugs alone and in combination were examined to provide a basis for the optimization of new dosing regimens. We present the following article in accordance with the MDAR reporting checklist (available at https://atm.amegroups.com/article/view/10.21037/atm-21$4595 / \mathrm{rc})$.

\section{Methods}

\section{Bacteria isolates}

The following 8 enterococci strains were studied: 5 vancomycin intrinsically resistant enterococci (No. 1 and No. 2 for E. gallinarum, and No. 3, No. 4, and No. 5 for E. casseliflavus), 1 vancomycin-resistant $E$. faecalis (No. 8), and 2 quality control strains (ATCC29212 and ATCC51299). No. 1 to No. 5 were provided by Tong Ren Hospital of Shanghai Jiao Tong University, and the remaining 3 strains were provided by The First Affiliated Hospital of the University of Science and Technology of China. Clinical isolates were isolated from bile, pus, and thoracoabdominal fluid, except for the quality control strains. All strains were identified using an automated VITEK-2 system (BioMerieux, Marcy l'Etoile, France). Notably, these strains were not isolated specifically for this study, but were part of routine laboratory procedures at the hospital.

\section{Antimicrobial agents and medium}

Linezolid, vancomycin, and fosfomycin were purchased from the National Institute for Food and Drug Control of China (Beijing, China). Mueller-Hinton agar (MHA; Oxoid, England) was used for the agar dilution method, culturing bacteria, and quantifying colony counts. MuellerHinton broth (MHB; Oxoid, England) was used for the broth microdilution method and bacterial culture.

\section{Vancomycin agar screening assay}

Vancomycin agar screening was used to test the susceptibility of the strains to vancomycin according to the Clinical and Laboratory Standards Institute (CLSI) (21). On brain heart infusion agar plates containing $6 \mathrm{mg} / \mathrm{L}$ vancomycin, 1-10 $\mu \mathrm{L}$ of $0.5 \mathrm{McF}$ arland bacterial suspension was added dropwise and incubated at $37^{\circ} \mathrm{C}$ for $24 \mathrm{~h}$. More than 1 colony or 1 layer of film growth was indicative of drug resistance. ATCC29212 was used as a sensitive control strain, and ATCC51299 was used as a resistant control strain. The experiment was replicated 3 times.

\section{Determination of the MIC}

According to the CLSI (22), the MICs of either linezolid or vancomycin were determined using the broth microdilution method. Put it briefly, a series of 2-fold dilutions of the antimicrobial drug were added to the small 96-well plates, followed by a 150 -fold dilution of the bacterial solution (approximately $1.5 \times 10^{8}$ colony-forming unit per milliliter, $\mathrm{CFU} / \mathrm{mL}$ ) in the logarithmic growth phase. The MIC of fosfomycin was determined using the agar dilution method. Specifically, a series of 2 -fold diluted antimicrobial drug agar plates (containing $25 \mathrm{mg} / \mathrm{L}$ of glucose 6-phosphate) were inoculated with $10^{4} \mathrm{CFU}$ per spot of bacteria. The plates were incubated at $37^{\circ} \mathrm{C}$ for $24 \mathrm{~h}$, and the results were observed at the lowest drug concentration without visible bacterial growth as MIC. ATCC29212 was used as a quality control strain. The experiment was repeated 3 times.

\section{Broth micro-checkerboard testing}

Eight drug dilutions were determined based on the MIC of the single drug, with the highest concentration being $2 \times$ MIC, in order of pairwise dilution. Starting from the lowerright corner of the 96-well plate, linezolid was added to each column from bottom to top at a concentration from largest to smallest ( $2 \times$ MIC-1/64× MIC), and fosfomycin was added to each row from right to left at a concentration from largest to smallest $(2 \times \mathrm{MIC}-1 / 64 \times \mathrm{MIC})$, and $25 \mathrm{mg} / \mathrm{L}$ of glucose 6-phosphate and $10^{6} \mathrm{CFU} / \mathrm{mL}$ of bacterial suspension were then added to each well. Both negative and positive controls were set up, and the plates were incubated at $37^{\circ} \mathrm{C}$ for $24 \mathrm{~h}$. The results were then observed. The fractional inhibition concentration index (FICI) was calculated using the following formula: FICI $=\mathrm{MIC}_{\mathrm{LNZ}+\mathrm{FOS}} /$ $\mathrm{MIC}_{\mathrm{LNZ}}+\mathrm{MIC}_{\mathrm{FOS}+\mathrm{LNZ}} / \mathrm{MIC}_{\mathrm{FOS}}$, where energy was defined as a FICI of $\leq 0.5$, and additivity was defined as a FICI of 
$>0.5$ but $<1$, indifference was defined as a FICI of $\geq 1$ but $<4$, while antagonism was defined as a FICI of $\geq 4$ (23). The experiment was repeated 3 times.

\section{Time-kill assays and factorial design}

Time-kill assays were performed according to guideline M26-A of the CLSI (24) with the following change: 3 strains (No. 2, No. 8, and ATCC29212) were selected based on the MIC and FICI results. The antimicrobial drug concentrations in the protocol were selected based on the MIC values and achievable levels in human serum, with linezolid $(1 / 4 \times, 1 \times$ MIC) and fosfomycin $(1 / 4 \times, 1 \times$ MIC) alone or in combination to form $8 \times 10 \mathrm{~mL}$ test tubes, and an additional growth control tube (without the antimicrobial drug). A final concentration of $25 \mathrm{mg} / \mathrm{L}$ of glucose 6-phosphate was added to the tubes containing fosfomycin with an initial inoculum of $5 \times 10^{5} \mathrm{CFU} / \mathrm{mL}$. The tubes were incubated at $37{ }^{\circ} \mathrm{C}$ with shaking, and sampled at 0,2 , $4,6,8,12$, and $24 \mathrm{~h}$, and the colonies were counted on the MHA plates. Based on the results of the two experiments, a 4-group trial of linezolid $(1 / 4 \times, 1 \times \mathrm{MIC})$ in combination with fosfomycin $\left(1 / 4 \times, 1 \times\right.$ MIC) was selected for the $2^{2}$ factorial design.

The bactericidal effect was determined by the change in the logarithmic value of the bacterial solution concentration at 24 versus $0 \mathrm{~h}$. The synergy of the combination was defined as a reduction in the colony count of $\geq 2 \log _{10} \mathrm{CFU} / \mathrm{mL}$ or more at $24 \mathrm{~h}$ for the combination compared to the most active single agent alone. The bactericidal effect was defined as a decrease in the colony count of $3 \log _{10} \mathrm{CFU} / \mathrm{mL}$ or more from the initial count (25).

\section{Induction and measurement of the PAE}

The PAE was measured using the method described previously (26). The PAEs of $1 \times$ MIC linezolid, $1 \times$ MIC fosfomycin alone, and their combination against the 3 strains described above were tested using $1 \times$ MIC as the drug concentration to which the strains were exposed in the time-kill assays. A starting inoculum of approximately $2 \times 10^{6} \mathrm{CFU} / \mathrm{mL}$ was obtained by adding bacteria from the exponential growth period to a $10-\mathrm{mL}$ test tube containing the corresponding antimicrobial drug (this point was recorded as moment $0 \mathrm{~h}$ ). After exposure to the antibacterial drug at $37^{\circ} \mathrm{C}$ for $2 \mathrm{~h}$, the antibiotics were removed with a dilution of 1:1,000 in a sterile broth (27), and the controls (unexposed to the antibacterial drug) were treated in a similar manner. Culture tubes with post-antibiotic stage bacteria and unexposed controls were incubated at $37^{\circ} \mathrm{C}$ for an additional 12 hours. The samples were extracted at 0 and $2 \mathrm{~h}$ (before and after dilution) and at 3, 4, 6, 8, 10, and $14 \mathrm{~h}$. Dilutions of each sample were inoculated onto the MHA plates and counted to determine the number of CFUs. The following formula for PAE was used (28): PAE = T $-\mathrm{C}$, where $\mathrm{T}$ is the time to increase the bacteria count by $1 \log _{10}$ $\mathrm{CFU} / \mathrm{mL}$ immediately after dilution in the cultures exposed to antibiotics, and $\mathrm{C}$ is the corresponding time for the controls.

\section{Measurement of the PASME and the SME}

The growth of bacteria at low concentrations of antimicrobial drugs was continued using $1 / 4 \times$ MIC as a sub-inhibitory concentration in the time-kill assays, thus simulating the decrease in concentration of antimicrobial drugs after being metabolized in vivo. To determine the PASME, the culture tubes in the post-antibiotic phase (selected for exposure to $1 \times$ MIC linezolid) were supplemented with $1 / 4 \times$ MIC linezolid, or $1 / 4 \times$ MIC fosfomycin (alone or in combination), and the cultures were prepared as described in the section titled, "Induction and measurement of the PAE," and re-incubated for $12 \mathrm{~h}$ at $37^{\circ} \mathrm{C}$. Samples were removed at 0 and $2 \mathrm{~h}$ (before and after dilution) and at 3, 4, 6, 8, 10, and $14 \mathrm{~h}$, and CFU values were determined as described in the PAE test section above. SME was measured from the tubes of control cultures that had not been exposed to antimicrobial drugs, after which 1/4× MIC linezolid, 1/4× MIC fosfomycin monotherapy, or their combinations were added, and the samples were incubated to determine the number of viable bacteria, similar to the determination of the PASME.

The following PASME formula was used (29): PASME $=T_{P A}-C$, where $T_{P A}$ is the time to increase the bacteria count by $1 \log _{10} \mathrm{CFU} / \mathrm{mL}$ immediately after dilution in the cultures that were exposed to antibiotics before and then to different sub-inhibitory concentrations, and $\mathrm{C}$ is the corresponding time for the controls that were not exposed. The PASME was chosen because in clinical situations in which intermittent antibiotic doses are used, the combined 
Table 1 Summary of MIC and FICI values of the 8 enterococci strains

\begin{tabular}{|c|c|c|c|c|c|}
\hline Strains & \multicolumn{3}{|c|}{ MIC (mg/L) } & $\frac{\mathrm{MIC}_{\text {combination }}}{\text { LNZ/FOS }}$ & $\frac{\mathrm{FICl}}{\mathrm{LNZ}+\mathrm{FOS}}$ \\
\hline No. 1 & 4 & 2 & 64 & $0.03125 / 64$ & 1.015 \\
\hline No. 2 & 4 & 2 & 128 & $0.5 / 32$ & 0.5 \\
\hline No. 3 & 2 & 2 & 128 & $1 / 32$ & 0.75 \\
\hline No. 5 & 1 & 4 & 128 & $1 / 32$ & 0.5 \\
\hline No. 8 & 128 & 2 & 128 & $1 / 32$ & 0.75 \\
\hline ATCC 29212 & 1 & 2 & 128 & $1 / 64$ & 1 \\
\hline ATCC 51299 & 256 & 2 & 128 & $0.5 / 32$ & 0.5 \\
\hline
\end{tabular}

VAN: $\leq 4 \mathrm{mg} / \mathrm{L}$, susceptible (S); 8-16 mg/L, intermediate (I); $\geq 32 \mathrm{mg} / \mathrm{L}$, resistant (R). LNZ: $\leq 2 \mathrm{mg} / \mathrm{L}$, susceptible (S); $4 \mathrm{mg} / \mathrm{L}$, intermediate (I); $\geq 8 \mathrm{mg} / \mathrm{L}$, resistant I. FOS: $\leq 64 \mathrm{mg} / \mathrm{L}$, susceptible (S); $128 \mathrm{mg} / \mathrm{L}$, intermediate (I); $\geq 256 \mathrm{mg} / \mathrm{L}$, resistant (R). MIC, minimum inhibitory concentration; VAN, vancomycin; LNZ, linezolid; FOS, fosfomycin; LNZ + FOS, linezolid-fosfomycin combination; FICI, fractional inhibitory concentration index.

effect of super-inhibitory and sub-inhibitory concentrations of a drug determines the time to the regeneration of the bacteria. The following SME formula was used (29): SME $=T_{S}-C$, where $T_{S}$ is the time to increase the bacteria count by $1 \log _{10} \mathrm{CFU} / \mathrm{mL}$ immediately after dilution in the cultures that were exposed only to the sub-inhibitory concentrations, and $\mathrm{C}$ is as defined as above.

\section{Statistical analysis}

GraphPad Prism software (version 8.0, GraphPad Software Inc., San Diego, CA, United States) was used for the statistical analysis. The main effects of the drug and drugdrug interactions were analyzed using a factorial design for the time-kill assays. A one-way analysis of variance (ANOVA) was used for the group comparisons. $\mathrm{P}<0.05$ indicated a statistically significant difference.

\section{Results}

\section{Antimicrobial susceptibility and broth micro-checkerboard testing}

In the vancomycin agar screening test, 7 strains, including ATCC51299, grew significantly on plates; however, ATCC29212 did not, which suggested that the selected clinical isolates were VRE strains. In the MIC test, according to the enterococcal MIC breakpoint (21), strains No. 1 to No. 5 were vancomycin-susceptible, No. 8 was vancomycin-resistant, and No. 4 was fosfomycin-resistant; none of the strains were linezolid resistant. The FICI values illustrated that linezolid combined with fosfomycin had a synergistic or an additive effect on the 6 strains, had an indifferent effect on the No. 1 and ATCC29212 strains, and did not have an antagonistic effect on any of the strains (see Table 1).

\section{Time-kill assays and factorial design}

For No. 2, No. 8, and ATCC29212, 1× MIC of fosfomycin caused the maximum decrease in bacterial load at 6 or $8 \mathrm{~h}$, and the $\log _{10} \mathrm{CFU} / \mathrm{mL}$ values decreased by $2.33 \pm 0.13$ $(6 \mathrm{~h}), 0.41 \pm 0.03(8 \mathrm{~h}), 2.95 \pm 0.05(8 \mathrm{~h})$, respectively. ATCC29212 even showed a bactericidal effect. However, in the next 12 hours, they all rebounded again, growing until they approached the control level. 1× MIC of linezolid maintained the time-kill curve against the three strains, showing bacteriostatic activity. 1/4× MIC of a single drug (linezolid or fosfomycin) had a minimal effect on the three strains (see Figure 1). The $\log _{10} \mathrm{CFU} / \mathrm{mL}$ values decreased by $1.83 \pm 0.12,0.64 \pm 0.04$, and $2.28 \pm 0.01$ at $24 \mathrm{~h}$ in the 2 -drug combination groups $(1 \times$ MIC linezolid combined with 1× MIC Fosfomycin), respectively. The 2-drug 


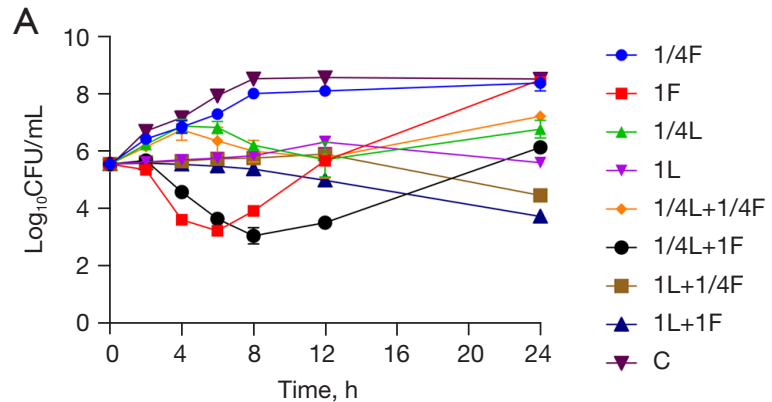

B
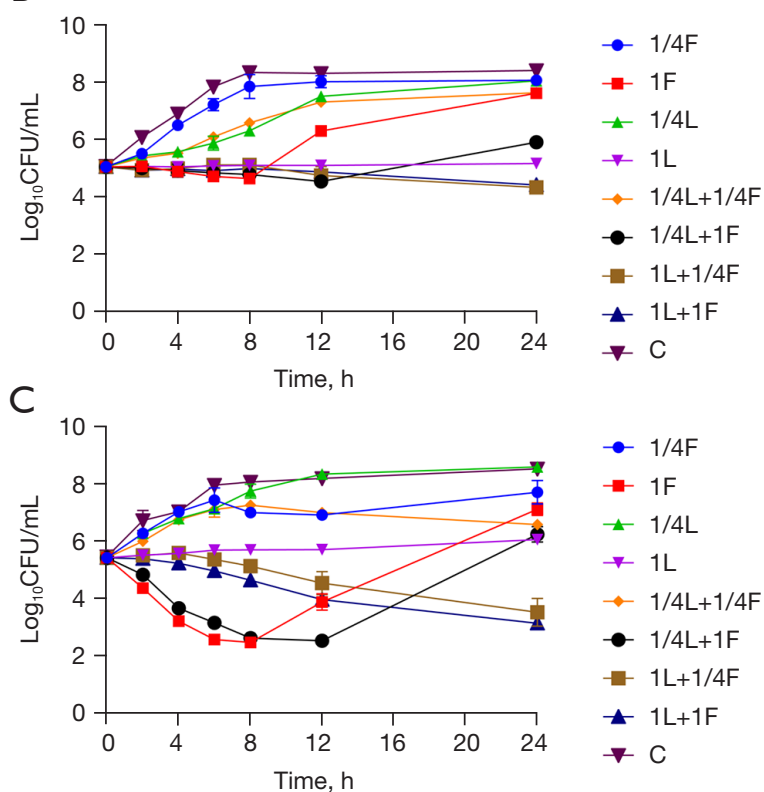

Figure 1 Time-kill curves of linezolid combined with fosfomycin alone or combined against three strains of Enterococcus, (A) vancomycin naturally resistant Enterococcus gallinarum (No. 2), (B) vancomycin-resistant Enterococcus faecium (No. 8), (C) vancomycin-susceptible Enterococcus faecalis (ATCC 29212), respectively. CFU, colony forming units; C, blank control; L, linezolid; F, fosfomycin; 1L, linezolid in $1 \times$ MIC, 1/4L , linezolid in $1 / 4 \times$ MIC, and so forth.

combinations had greater synergistic effects on No. 2 and ATCC29212 than the most effective single-drug group. Notably, when linezolid $(1 \times \mathrm{MIC})$ was combined with subinhibitory concentrations of fosfomycin (1/4× MIC), the $\log _{10} \mathrm{CFU} / \mathrm{mL}$ values of the 3 strains also decreased to varying degrees at $24 \mathrm{~h}$ to $1.10 \pm 0.03,0.72 \pm 0.02$, and $1.89 \pm 0.46$, respectively. This combination had a synergistic effect on ATCC29212 (see Table 2).

In the $2^{2}$ factorial design of linezolid $(1 / 4 \times, 1 \times$ MIC) combined with fosfomycin $(1 / 4 \times, 1 \times$ MIC) (see Table 3), there was a statistically significant interaction $(\mathrm{P}<0.05)$ between the combination of the two drugs on the change in the logarithmic values of No. 2 and No. 8 in the $24 \mathrm{~h}$ colony count, and the change in the MICs of both drugs also had an effect on the change in the colony count at $24 \mathrm{~h}$ $(\mathrm{P}<0.05)$. For ATCC29212, the interaction of the 2-drug combination was not statistically significant $(\mathrm{P}>0.05)$, and the difference between linezolid combined with different MICs of fosfomycin on the change in log colony count at $24 \mathrm{~h}$ was not statistically significant $(\mathrm{P}>0.05)$, but linezolid exerted the main effect $(\mathrm{P}<0.05$; see Table 3$)$. The one-way ANOVA showed that the difference between $1 \times$ MIC of linezolid combined with two concentrations of fosfomycin $(1 / 4 \times, 1 \times$ MIC) on No. 8 and ATCC29212 in the change in $\log$ colony number values at $24 \mathrm{~h}$ was not statistically significant ( $\mathrm{P}>0.05$; see Figure 2$)$.

\section{PAEs, PASMEs, and SMEs}

The mean values $[ \pm$ standard deviation $(\mathrm{SD})]$ and regrowth curves for the PAE, PASME, and SME for No. 2, No. 8, and ATCC29212 are shown in Table 4 and Figure 3, respectively. Linezolid and fosfomycin at $1 \times$ MIC produced a PAE against all three strains. There was no statistically significant difference in the PAE between the two drug combinations with that of linezolid alone $(\mathrm{P}>0.05$; see Figure 4$)$; however, as Table 4 shows, the duration of PAE was longer in both drug combination groups than in the single-drug groups. The SME for the three strains with $1 / 4 \times$ MIC of linezolid and fosfomycin was similar to that of the PAE described above. For No. 8, there was no statistically significant decrease in the SME after the combination of the two drugs compared with linezolid alone $(\mathrm{P}>0.05$; see Figure 4). Additionally, 1/4× MIC of linezolid combined with $1 / 4 \times$ MIC of fosfomycin significantly prolonged the duration of the PASME compared to that of a single drug for all three strains $(\mathrm{P}<0.05$; see Figure 4$)$ (the values were $1.97 \pm 0.01,4.32 \pm 0.18$, and $1.74 \pm 0.13 \mathrm{~h}$, respectively). As Table 4 shows, the duration of the PASME was much longer than the PAE and the SME. The representative images in Figure 5 show the variation of colony counts in the timekill assays and in the measurement of PAE, PASME, and 
Table 2 Changes in the logarithmic values at $24 v s .0 \mathrm{~h}$ in the bacterial solution concentrations

\begin{tabular}{lcccccccccc}
\hline \multirow{2}{*}{ Strains } & \multicolumn{7}{c}{$\Delta \log _{10}(\mathrm{CFU} / \mathrm{mL})=\log _{10}\left(\mathrm{CFU}_{24 / \mathrm{mL}}\right)-\log _{10}\left(\mathrm{CFU}_{0 / \mathrm{mL}}\right)$} \\
\cline { 2 - 9 } & $1 / 4 \mathrm{~F}$ & $1 \mathrm{~F}$ & $1 / 4 \mathrm{~L}$ & $1 \mathrm{~L}$ & $1 / 4 \mathrm{~L}+1 / 4 \mathrm{~F}$ & $1 / 4 \mathrm{~L}+1 \mathrm{~F}$ & $1 \mathrm{~L}+1 / 4 \mathrm{~F}$ & $1 \mathrm{~L}+1 \mathrm{~F}$ & $\mathrm{C}$ \\
\hline No. 2 & $2.82 \pm 0.28$ & $2.93 \pm 0.01$ & $1.21 \pm 0.31$ & $0.05 \pm 0.13$ & $1.67 \pm 0.05$ & $0.58 \pm 0.05$ & $-1.10 \pm 0.03$ & $-1.83 \pm 0.12$ & $3.04 \pm 0.01$ \\
No. 8 & $3.02 \pm 0.24$ & $2.57 \pm 0.11$ & $3.00 \pm 0.29$ & $0.11 \pm 0.05$ & $2.58 \pm 0.18$ & $0.86 \pm 0.15$ & $-0.72 \pm 0.02$ & $-0.64 \pm 0.04$ & $3.33 \pm 0.14$ \\
ATCC29212 & $2.29 \pm 0.38$ & $1.70 \pm 0.19$ & $3.17 \pm 0.03$ & $0.64 \pm 0.01$ & $1.16 \pm 0.04$ & $0.82 \pm 0.29$ & $-1.89 \pm 0.46$ & $-2.28 \pm 0.01$ & $3.07 \pm 0.03$ \\
\hline
\end{tabular}

All data are presented as mean \pm SD. CFU, colony forming units; C, blank control; L, linezolid; F, fosfomycin; 1L, linezolid in 1× MIC, 1/4L, linezolid in $1 / 4 \times \mathrm{MIC}$, and so forth.

Table 3 The factorial design and analysis results of logarithmic changes of linezolid $(1 / 4 \times, 1 \times$ MIC) $\times$ fosfomycin $(1 / 4 \times, 1 \times$ MIC) bacteria concentration at 24 vs. $0 \mathrm{~h}$

\begin{tabular}{|c|c|c|c|c|c|c|c|c|c|c|c|}
\hline Strains & $\triangle \log _{10} \mathrm{CFU}$ & \multicolumn{2}{|c|}{$1 / 4 \mathrm{~L}$} & \multicolumn{2}{|c|}{$1 \mathrm{~L}$} & ANOVA table & SS & DF & MS & $\mathrm{F}(\mathrm{DFn}, \mathrm{DFd})$ & $P$ value \\
\hline \multirow[t]{3}{*}{ No. 2} & $1 / 4 \mathrm{~F}$ & 1.64 & 1.70 & -1.07 & -1.12 & Interaction & 0.06216 & 1 & 0.06216 & $F(1,4)=12.28$ & 0.0248 \\
\hline & & & & & & Row factor & 1.657 & 1 & 1.657 & $F(1,4)=327.3$ & $<0.0001$ \\
\hline & $1 \mathrm{~F}$ & 0.55 & 0.62 & -1.92 & -1.75 & Column factor & 13.4 & 1 & 13.4 & $F(1,4)=2647$ & $<0.0001$ \\
\hline \multirow[t]{4}{*}{ No. 8} & $1 / 4 \mathrm{~F}$ & 2.71 & 2.46 & -0.73 & -0.71 & Interaction & 1.632 & 1 & 1.632 & $F(1,4)=119.8$ & 0.0004 \\
\hline & & & & & & Row factor & 1.351 & 1 & 1.351 & $F(1,4)=99.15$ & 0.0006 \\
\hline & $1 \mathrm{~F}$ & 0.96 & 0.75 & -0.66 & -0.60 & Column factor & 11.47 & 1 & 11.47 & $F(1,4)=841.6$ & $<0.0001$ \\
\hline & & & & & & Residual & 0.0545 & 4 & 0.01363 & & \\
\hline \multirow{2}{*}{ ATCC29212 } & $1 \mathrm{~F}$ & 1.03 & 0.61 & -2.29 & -2.27 & Column factor & 18.95 & 1 & 18.95 & $F(1,4)=252.7$ & $<0.0001$ \\
\hline & & & & & & Residual & 0.2999 & 4 & 0.07498 & & \\
\hline
\end{tabular}

$\Delta \log _{10}(\mathrm{CFU} / \mathrm{mL})=\log _{10}\left(\mathrm{CFU}_{24 / \mathrm{mL}}\right)-\log _{10}(\mathrm{CFU} / \mathrm{mL})$. CFU, colony forming units; G, group; L, linezolid; F, fosfomycin; $1 \mathrm{~L}$, linezolid in 1× MIC, $1 / 4 \mathrm{~L}$, linezolid in $1 / 4 \times \mathrm{MIC}$, and so forth.

SME assays.

\section{Discussion}

The resistance phenotypes of VRE fall into two categories, 1 with high levels of vancomycin resistance (VanA, B, and D), and the other with low resistance to vancomycin (VanC, E, and G) (5). High levels of resistance associated with the acquisition genes are most common in E. faecalis and $E$. faecium isolates. Conversely, E. gallinarum and E. casseliflavus are intrinsically resistant to low levels of vancomycin (30). In this study, both these resistant types were selected from the clinical laboratory, and antimicrobial susceptibility testing showed that strains No. 1 to No. 5 were vancomycin low-level resistant, while No. 8 was vancomycin-resistant to E. faecalis. The strains showed varying degrees of resistance to vancomycin. In recent years, potential treatment options have included linezolid and fosfomycin $(3,31,32)$.

The synergistic effect of linezolid combined with fosfomycin against Staphylococcus aureus and enterococci has been reported previously $(14,33,34)$. We chose different types of enterococci in the present study; however, the results of FICI for the 2-drug combination are similar to those of Qi et al. (14). The results all suggested that the 

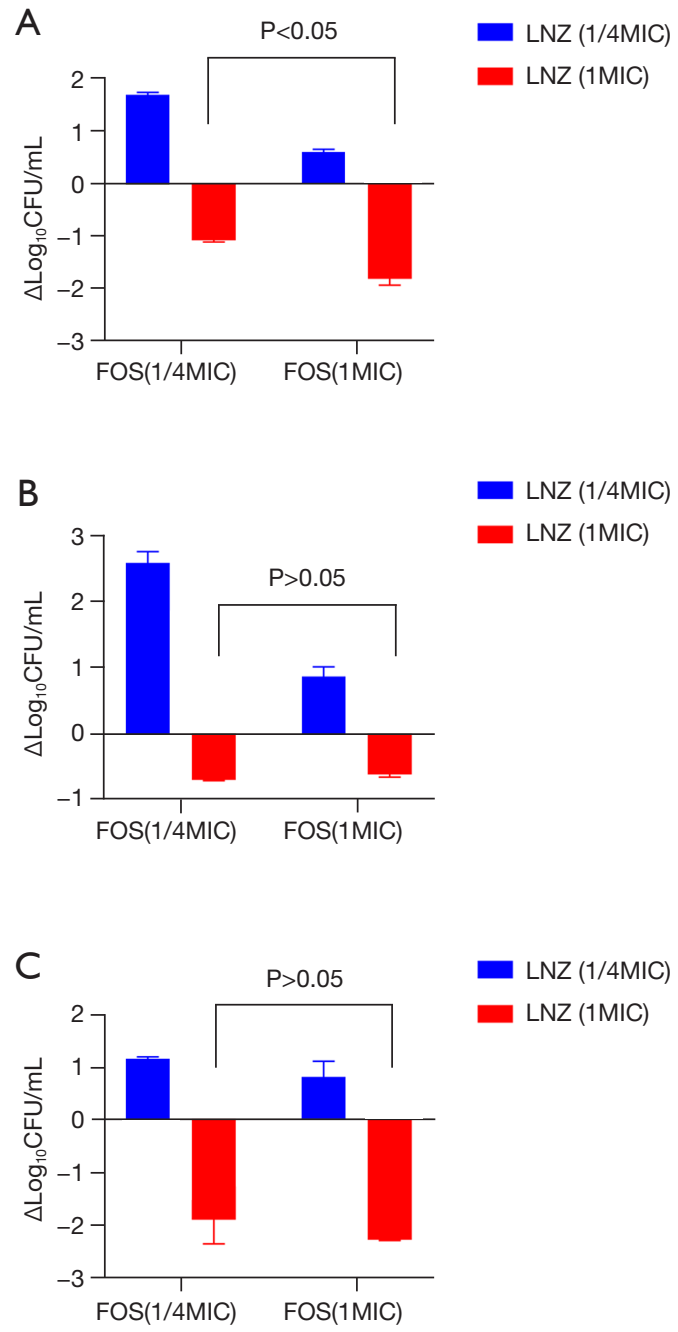

Figure 2 The factorial design and one-way ANOVA of logarithmic changes of linezolid $(1 / 4 \times, 1 \times$ MIC) $\times$ fosfomycin $(1 / 4 \times, 1 \times$ MIC) against three strains of Enterococcus at $24 v s$. $0 \mathrm{~h},(\mathrm{~A})$ vancomycin naturally resistant Enterococcus gallinarum (No. 2), (B) vancomycin-resistant Enterococcus faecium (No. 8), (C) vancomycin-susceptible Enterococcus faecalis (ATCC 29212), respectively. CFU, colony forming units; LNZ, linezolid; FOS, fosfomycin.

2-drug combination has a synergistic or an additive effect against VREs, including vancomycin low-level resistant strains. Unlike the checkerboard method, which simply provides susceptibility data, time-kill assays are more appropriate for clinical situations that require bactericidal therapy, as these assays measure the antimicrobial activity of drug combinations (35). In the present study, to further investigate whether there was an interaction between linezolid and fosfomycin, we conducted a factorial analysis based on the results of the time-kill assays (20). The results of the time-kill assays differed somewhat to those of the checkerboard method. Specifically, the combination of the two antibiotics had a synergistic effect against No. 8 in the checkerboard method that was not observed in the timekill assays. Unsurprisingly, experiments of drug interactions can lead to opposite conclusions if different methods are adopted (36). Notably, we found that the difference in the bactericidal effect against No. 8 at $24 \mathrm{~h}$ was not very large $(\mathrm{P}>0.05)$ when high concentrations of linezolid $(1 \times \mathrm{MIC})$ were combined with high and low concentrations of fosfomycin $(1 / 4 \times, 1 \times$ MIC). This may be because fosfomycin only exerts an antimicrobial effect in the initial phase (see Figures 1,2). In the $2^{2}$ factorial design, both linezolid and fosfomycin interacted with 2 VREs $(\mathrm{P}<0.05)$, while linezolid had a major effect on the sensitive quality control strain (ATCC29212) (see Table 3). Thus, linezolid can be combined with fosfomycin to target vancomycinresistant strains, but linezolid monotherapy is sufficient to target sensitive strains.

Pharmacodynamic parameters, such as the PAE, PASME, and SME, are important guides for describing antimicrobial activity and determining the optimal dosing regimen (37). To date, no other studies appear to have been conducted on the PAE of linezolid in combination with fosfomycin on enterococci, and any studies have only examined the PAE of linezolid alone. Hosgor-Limoncu et al. found that the PAE of linezolid against $E$. faecalis was slightly higher than that of other strains after a $1 \mathrm{~h}$ exposure to $1 \times$ MIC linezolid, and the in vitro PAE against enterococci was $1.1-1.4 \mathrm{~h}(38,39)$.

When previous studies have shown the combined significance of linezolid and fosfomycin on VREs, it is then of clinical relevance to explore co-administration regimens through studies of antibiotic-related effects. We first assessed the PAE of a 2-drug combination against VREs, and showed that each single drug exerted some degree of PAE on the strains, but the 2-drug combination prolonged the duration of the PAE (see Table 4). However, for the three different types of enterococci, the PAE after the 
Table 4 Results of linezolid combined with fosfomycin on the PAE, PASME, and SME of 3 strains

\begin{tabular}{|c|c|c|c|c|c|c|c|c|c|}
\hline Strains & \multicolumn{3}{|c|}{ PAE } & \multicolumn{3}{|c|}{ PASME } & \multicolumn{3}{|c|}{ SME } \\
\hline No. 2 & $0.22 \pm 0.06$ & $0.47 \pm 0.05$ & $0.55 \pm 0.01$ & $0.54 \pm 0.07$ & $1.16 \pm 0.03$ & $1.97 \pm 0.01$ & $0.17 \pm 0.03$ & $0.73 \pm 0.18$ & $1.08 \pm 0.02$ \\
\hline No. 8 & $0.14 \pm 0.01$ & $0.77 \pm 0.14$ & $1.15 \pm 0.23$ & $0.28 \pm 0.02$ & $3.16 \pm 0.24$ & $4.32 \pm 0.18$ & $0.20 \pm 0.01$ & $2.60 \pm 0.03$ & $2.41 \pm 0.18$ \\
\hline ATCC29212 & $0.24 \pm 0.02$ & $0.59 \pm 0.10$ & $0.66 \pm 0.01$ & $0.37 \pm 0.01$ & $1.37 \pm 0.03$ & $1.74 \pm 0.13$ & $0.28 \pm 0.02$ & $0.91 \pm 0.24$ & $1.27 \pm 0.01$ \\
\hline
\end{tabular}

All data are presented as mean \pm SD. PAE, post-antibiotic effect; PASME, post-antibiotic sub-MIC effect; SME, sub-MIC effect; L, linezolid; F, fosfomycin; $1 \mathrm{~L}$, linezolid in $1 \times \mathrm{MIC}, 1 / 4 \mathrm{~L}$, linezolid in 1/4× MIC, and so forth.

A
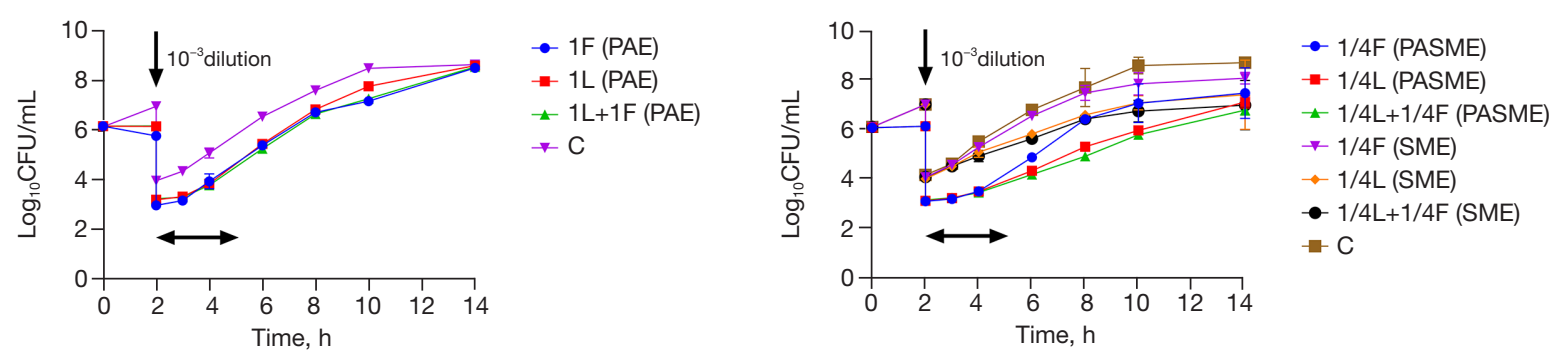

B
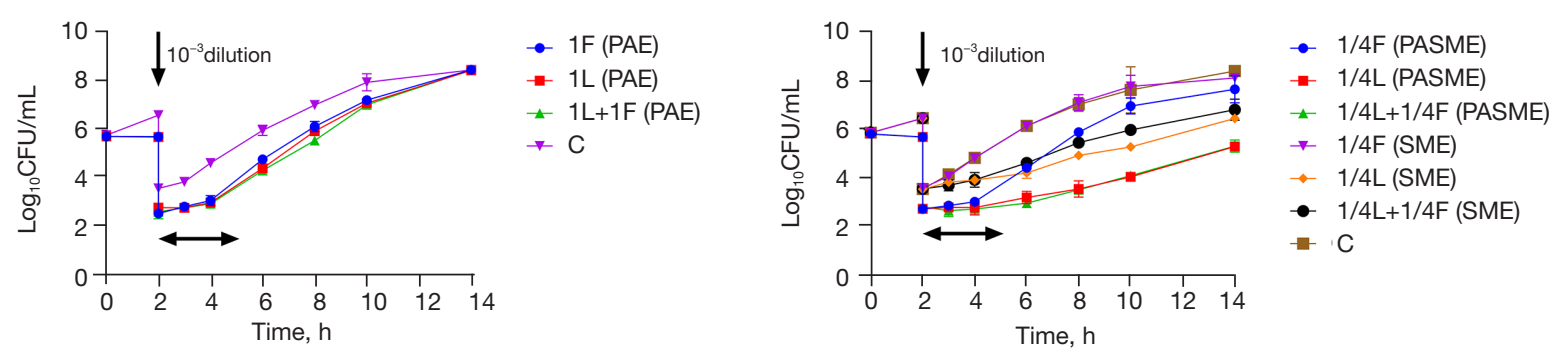

C
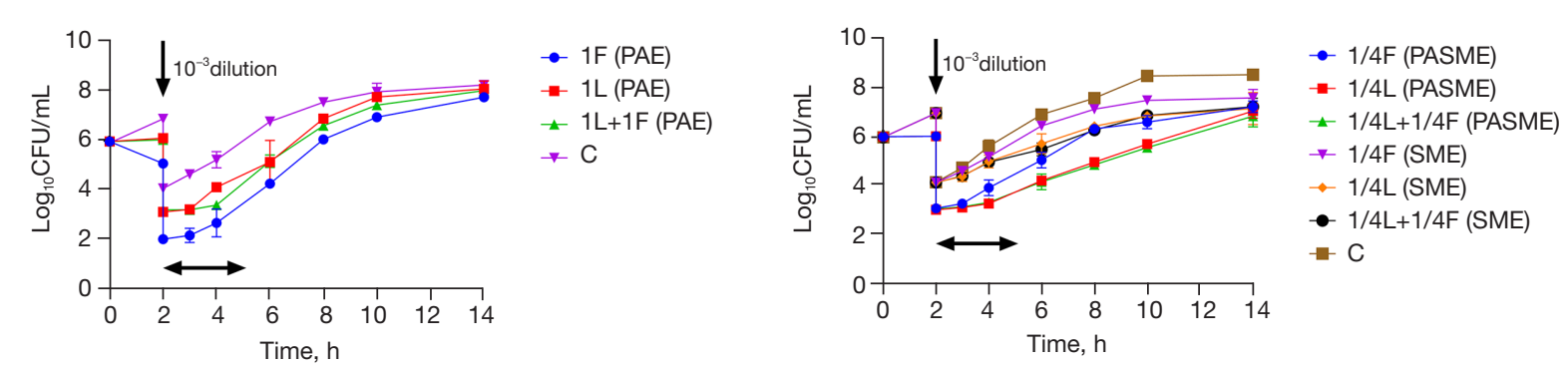

Figure 3 Growth curve of linezolid combined with fosfomycin on PAE, PASME and SME of three strains, (A) vancomycin naturally resistant Enterococcus gallinarum (No. 2), (B) vancomycin-resistant Enterococcus faecium (No. 8), (C) vancomycin-susceptible Enterococcus faecalis (ATCC 29212), respectively. CFU, colony forming units; C, blank control; L, linezolid; F, fosfomycin; 1/4L, linezolid

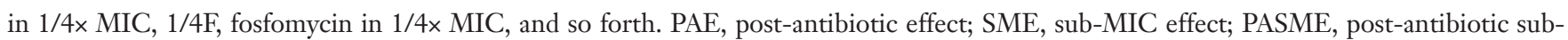
MIC effect. 


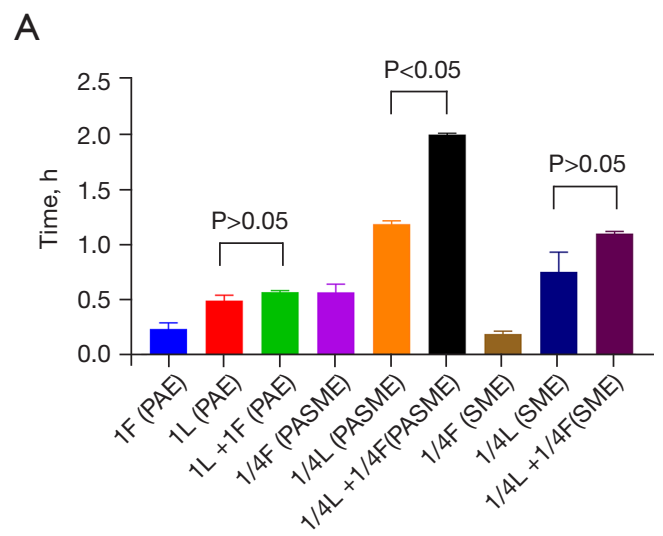

B

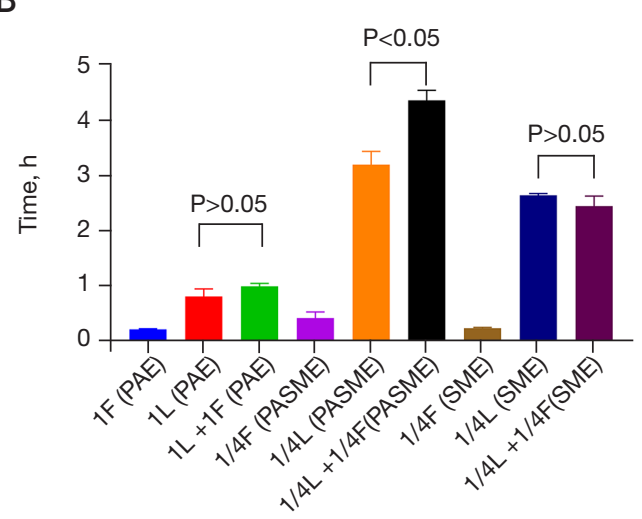

C

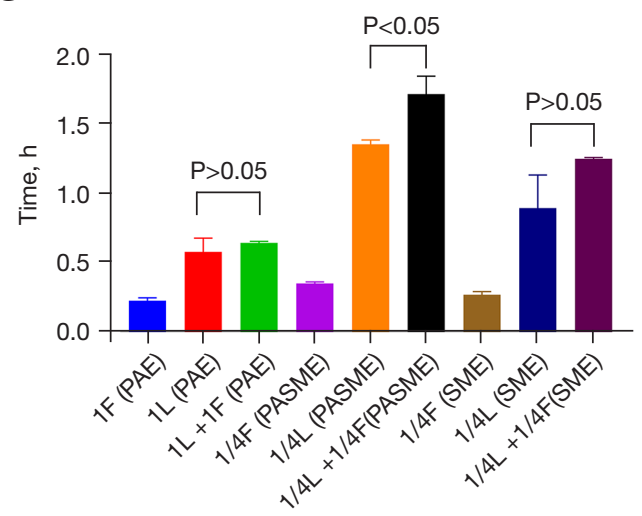

Figure 4 One-way ANOVA analysis of linezolid combined with fosfomycin on PAE, PASME and SME of 3 strains, (A) vancomycin naturally resistant Enterococcus gallinarum (No. 2), (B) vancomycin-resistant Enterococcus faecium (No. 8), (C) vancomycin-susceptible Enterococcus faecalis (ATCC 29212), respectively. L, linezolid; F, fosfomycin; 1L, linezolid in 1× MIC, $1 / 4 \mathrm{~L}$, linezolid in 1/4× MIC, and so forth. PAE, post-antibiotic effect; SME, sub-MIC effect; PASME, post-antibiotic sub-MIC effect. combination was not significantly elevated compared to that of linezolid alone $(\mathrm{P}>0.05)$, but was significantly different compared to that of fosfomycin alone $(\mathrm{P}<0.05)$. When the effect of exposure to high drug concentrations was not considered and only the growth of the strains at the SME of the drug was studied, the situation was similar to that of the PAE; that is, the difference in the SMEs for the 2-drug combination was not statistically significant compared to that of linezolid monotherapy $(\mathrm{P}>0.05)$. This may be related to the fact that linezolid exerts a bacteriostatic effect, while fosfomycin exerts a bactericidal effect in the early stage $(40,41)$.

In clinical practice, the entire duration of the dosing interval of antimicrobial drugs includes both the time when the drug concentration is above the MIC and the PAE, as the sub-inhibitory concentration of the drug will always follow the super-inhibitory concentration as it is metabolized in vivo (37). Since sub-inhibitory concentrations may be present after therapeutic dosing, the PASME better reflects the true in vivo situation than the PAE (42). In relation to the 2-drug combination, we only examined 1 scenario in which a strain was exposed to $1 \times$ MIC of linezolid; however, we found that the duration of the PASME was significantly more prolonged $(\mathrm{P}<0.05)$ for the 2 -drug combination at sub-inhibitory concentrations compared to the single drug. Thus, if fosfomycin has little effect on the PAE and SME in a 2-drug combination, a sub-inhibitory concentration of fosfomycin can be considered in clinical settings to prolong the PASME by administering it again $2 \mathrm{~h}$ after linezolid administration. This, in combination with the small and inexpensive fosfomycin, allows for a longer dosing interval for linezolid, thereby reducing the use of the more expensive linezolid in the regimen, while reducing the risk of screening for linezolid-resistant strains. However, further clinical trials need to be conducted to confirm these results.

In conclusion, our findings suggest that linezolid in combination with fosfomycin not only has a synergistic or additive effect but also an interactive effect against VREs, and that linezolid can be selected for a combination regimen design with sub-inhibitory concentrations of fosfomycin. Studies of the PAE, PASME, and SME with the 2-drug combination found that the continued administration of sub-inhibitory concentrations of fosfomycin after $2 \mathrm{~h}$ of linezolid administration prolonged the duration of the 

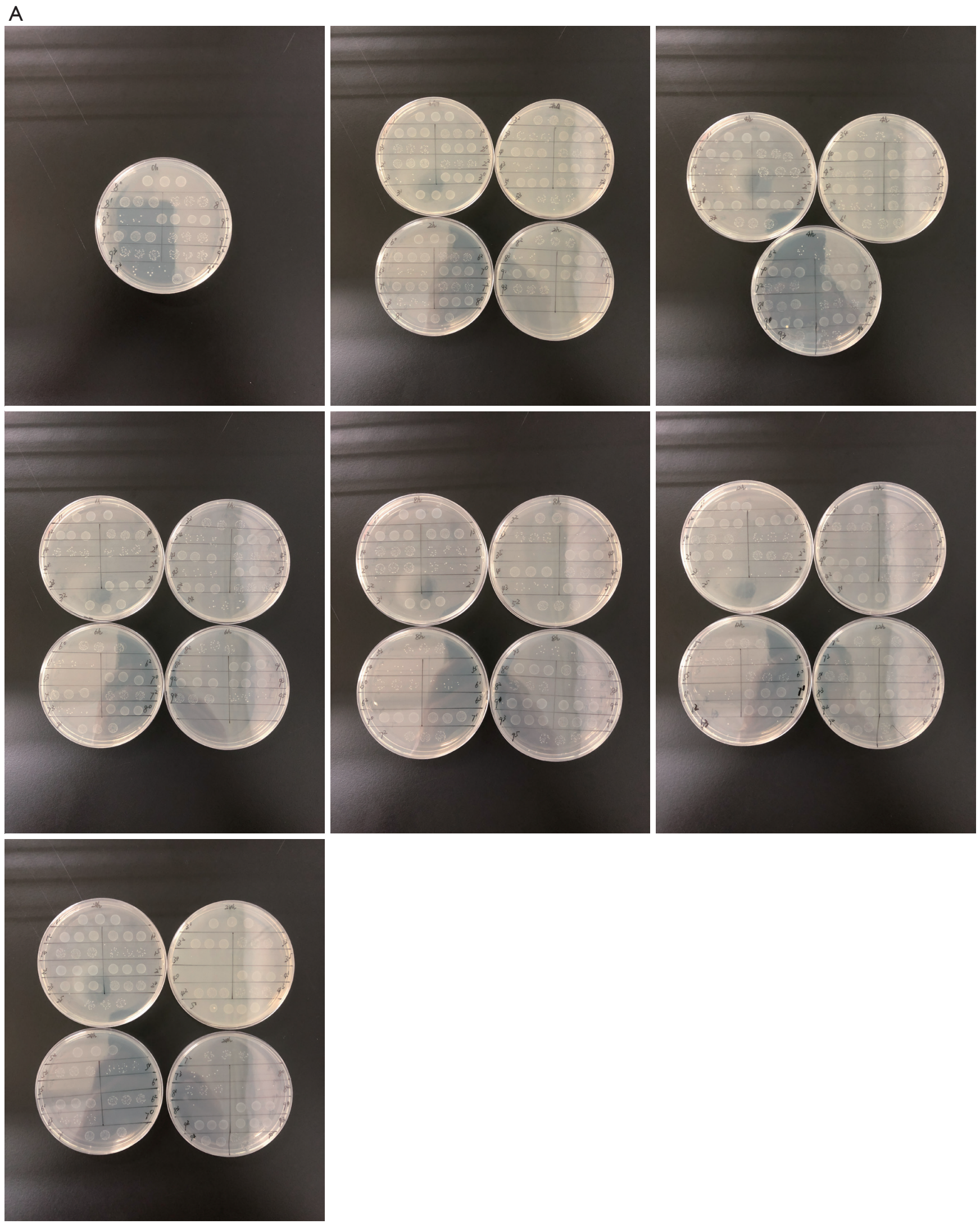
B
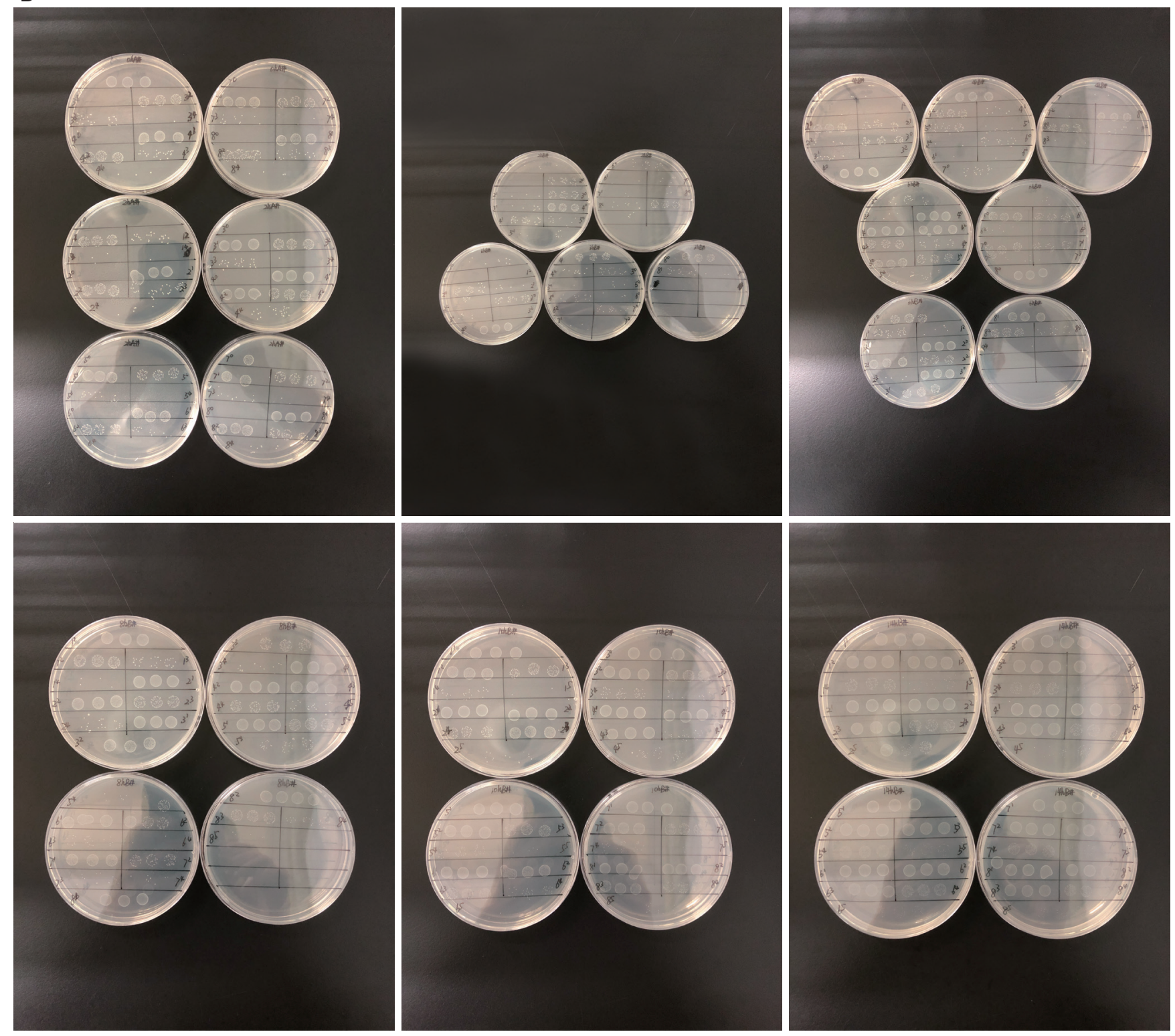

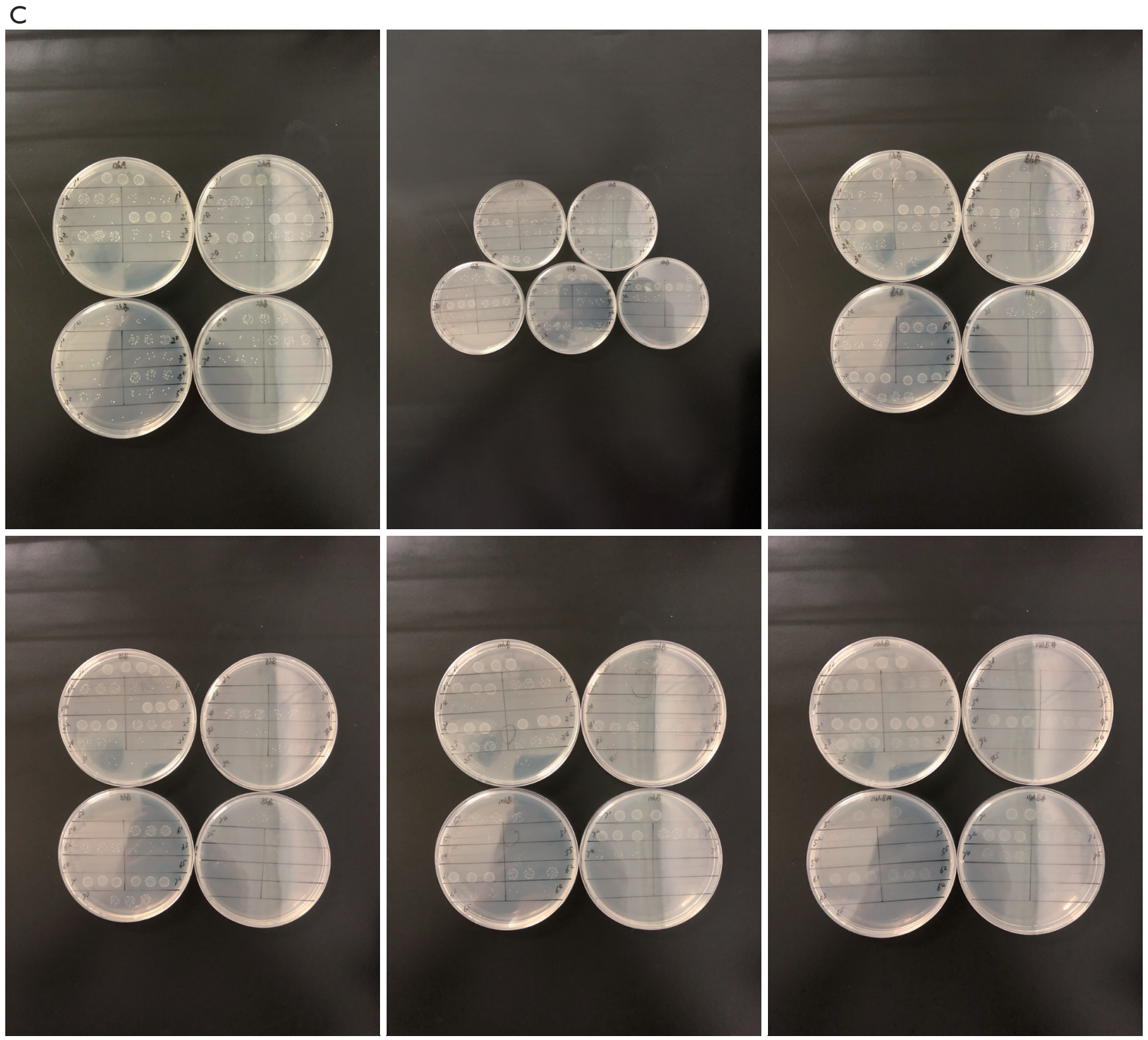

Figure 5 Representative images of colony formation. (A) Time-kill assays for strain No. 2, (B) measurement of the PAE for stain No. 8 , (C) measurement of the PASME and the SME for stain No. 8, respectively.

PASME. Thus, we believe that when linezolid is clinically selected for the treatment of VRE infections, sub-inhibitory concentrations of fosfomycin can be administered at multiple intervals to improve the therapeutic effect.

\section{Acknowledgments}

Funding: This study was supported by the National Natural Science Foundation of China (81173133), the Fund of Excellent Talents in Colleges and Universities of Anhui 
Province, China (gxbjZD06), and the Fund for Academic Leaders of Anhui Province, China (2015D068).

\section{Footnote}

Reporting Checklist: The authors have completed the MDAR reporting checklist. Available at https://atm.amegroups. com/article/view/10.21037/atm-21-4595/rc

Data Sharing Statement: Available at https://atm.amegroups. com/article/view/10.21037/atm-21-4595/dss

Conflicts of Interest: All authors have completed the ICMJE uniform disclosure form (available at https://atm. amegroups.com/article/view/10.21037/atm-21-4595/coif). The authors have no conflicts of interest to declare.

Ethical Statement: The authors are accountable for all aspects of the work, including ensuring that any questions related to the accuracy or integrity of any part of the work have been appropriately investigated and resolved.

Open Access Statement: This is an Open Access article distributed in accordance with the Creative Commons Attribution-NonCommercial-NoDerivs 4.0 International License (CC BY-NC-ND 4.0), which permits the noncommercial replication and distribution of the article with the strict proviso that no changes or edits are made and the original work is properly cited (including links to both the formal publication through the relevant DOI and the license). See: https://creativecommons.org/licenses/by-nc-nd/4.0/.

\section{References}

1. Uttley AH, Collins $\mathrm{CH}, \mathrm{Naidoo}$ J, et al. Vancomycinresistant enterococci. Lancet 1988;1:57-8.

2. Leclercq R, Derlot E, Duval J, et al. Plasmid-mediated resistance to vancomycin and teicoplanin in Enterococcus faecium. N Engl J Med 1988;319:157-61.

3. Wang JL, Hsueh PR. Therapeutic options for infections due to vancomycin-resistant enterococci. Expert Opin Pharmacother 2009;10:785-96.

4. Zeng D, Debabov D, Hartsell TL, et al. Approved Glycopeptide Antibacterial Drugs: Mechanism of Action and Resistance. Cold Spring Harb Perspect Med 2016;6:a026989.

5. Courvalin P. Vancomycin resistance in gram-positive cocci. Clin Infect Dis 2006;42 Suppl 1:S25-34.
6. Mendes RE, Castanheira M, Farrell DJ, et al. Longitudinal (2001-14) analysis of enterococci and VRE causing invasive infections in European and US hospitals, including a contemporary (2010-13) analysis of oritavancin in vitro potency. J Antimicrob Chemother 2016;71:3453-8.

7. Reyes K, Bardossy AC, Zervos M. Vancomycin-Resistant Enterococci: Epidemiology, Infection Prevention, and Control. Infect Dis Clin North Am 2016;30:953-65.

8. Isenman H, Fisher D. Advances in prevention and treatment of vancomycin-resistant Enterococcus infection. Curr Opin Infect Dis 2016;29:577-82.

9. O'Driscoll T, Crank CW. Vancomycin-resistant enterococcal infections: epidemiology, clinical manifestations, and optimal management. Infect Drug Resist 2015;8:217-30.

10. Yim J, Smith JR, Rybak MJ. Role of Combination Antimicrobial Therapy for Vancomycin-Resistant Enterococcus faecium Infections: Review of the Current Evidence. Pharmacotherapy 2017;37:579-92.

11. Rybak JM, Barber KE, Rybak MJ. Current and prospective treatments for multidrug-resistant gram-positive infections. Expert Opin Pharmacother 2013;14:1919-32.

12. Bender JK, Cattoir V, Hegstad K, et al. Update on prevalence and mechanisms of resistance to linezolid, tigecycline and daptomycin in enterococci in Europe: Towards a common nomenclature. Drug Resist Updat 2018;40:25-39.

13. Múñez Rubio E, Ramos Martínez A, Fernández Cruz A. Fosfomycin in antimicrobial stewardship programs. Rev Esp Quimioter 2019;32 Suppl 1:62-6.

14. Qi C, Xu S, Wu M, et al. Pharmacodynamics Of Linezolid-Plus-Fosfomycin Against VancomycinSusceptible And -Resistant Enterococci In Vitro And In Vivo Of A Galleria mellonella Larval Infection Model. Infect Drug Resist 2019;12:3497-505.

15. Jiang L, Xie N, Chen M, et al. Synergistic Combination of Linezolid and Fosfomycin Closing Each Other's Mutant Selection Window to Prevent Enterococcal Resistance. Front Microbiol 2021;11:605962.

16. Craig W. Pharmacodynamics of antimicrobial agents as a basis for determining dosage regimens. Eur J Clin Microbiol Infect Dis 1993;12 Suppl 1:S6-8.

17. Cars O, Odenholt-Tornqvist I. The post-antibiotic subMIC effect in vitro and in vivo. J Antimicrob Chemother 1993;31 Suppl D:159-66.

18. Odenholt-Tornqvist I, Löwdin E, Cars O. Postantibiotic effects and postantibiotic sub-MIC effects of roxithromycin, clarithromycin, and azithromycin on 
respiratory tract pathogens. Antimicrob Agents Chemother 1995;39:221-6.

19. Haerling Adamson K, Prion S. Two-by-Two Factorial Design. Clin Simul Nurs 2020;49:90-1.

20. McFarland MM, Scott EM, Li Wan Po A. Time-survival studies for quantifying effects of azlocillin and tobramycin on Pseudomonas aeruginosa. Antimicrob Agents Chemother 1994;38:1271-6.

21. CLSI. Performance Standards for Antimicrobial Susceptibility Testing. 29th ed. CLSI supplement M100.Wayne, PA: Clinical and Laboratory Standards Institute, 2019.

22. CLSI. Methods for Dilution Antimicrobial Susceptibility Tests for Bacteria That Grow Aerobically.11th ed. CLSI standard M07. Wayne, PA: Clinical and Laboratory Standards Institute, 2018.

23. Lewis RE, Diekema DJ, Messer SA, et al. Comparison of Etest, chequerboard dilution and time-kill studies for the detection of synergy or antagonism between antifungal agents tested against Candida species. J Antimicrob Chemother 2002;49:345-51.

24. National Committee of Clinical Laboratory Standards. Methods for Determining Bactericidal Activity of Antimicrobial Agents: Approved Guideline M26-A. NCCLS, Wayne, USA, 1999.

25. Aktas G. In Vitro Synergistic Effect of Vancomycin Combined with Daptomycin Against VancomycinResistant Enterococci. Microb Drug Resist 2019;25:1484-9.

26. Craig WA, Gudmundsson S. Postantibiotic effect. In: Lorian V. editor. Antibiotics in Laboratory Medicine. Baltimore, MD: Williams and Wilkins Co., 1996:296-329.

27. Bundtzen RW, Gerber AU, Cohn DL, et al. Postantibiotic suppression of bacterial growth. Rev Infect Dis 1981;3:28-37.

28. Craig, WA, Gudmundsson S. The postantibiotic effect. In Lorian V. editor. Antibiotics in laboratory medicine. Baltimore: The Williams \& Wilkins Co., 1991:403-31.

29. Odenholt-Tornquist I, Löwdin E, Cars O. Pharmacodynamic effects of subinhibitory concentrations of beta-lactam antibiotics in vitro. Antimicrob Agents Chemother 1991;35:1834-9.

30. Watanabe S, Kobayashi N, Quiñones D, et al. Genetic diversity of the low-level vancomycin resistance gene vanC-2/vanC-3 and identification of a novel vanC subtype (vanC-4) in Enterococcus casseliflavus. Microb Drug Resist 2009;15:1-9.
31. Allerberger F, Klare I. In-vitro activity of fosfomycin against vancomycin-resistant enterococci. J Antimicrob Chemother 1999;43:211-7.

32. Mercuro NJ, Davis SL, Zervos MJ, et al. Combatting resistant enterococcal infections: a pharmacotherapy review. Expert Opin Pharmacother 2018;19:979-92.

33. Chai D, Liu X, Wang R, et al. Efficacy of Linezolid and Fosfomycin in Catheter-Related Biofilm Infection Caused by Methicillin-Resistant Staphylococcus aureus. Biomed Res Int 2016;2016:6413982.

34. Chen H, Li L, Liu Y, et al. In vitro activity and postantibiotic effects of linezolid in combination with fosfomycin against clinical isolates of Staphylococcus aureus. Infect Drug Resist 2018;11:2107-15.

35. Eliopoulos GM, Moellering RC Jr, Pillai SK. Antimicrobial combinations. In: LorianV. editor. Antibiotics in laboratory medicine. Philadelphia: Lippincott Williams and Wilkins, 2005:365-440.

36. Odds FC. Synergy, antagonism, and what the chequerboard puts between them. J Antimicrob Chemother 2003;52:1.

37. Lee SY. Postantibiotic effects and postantibiotic subMIC effects of amoxicillin on Streptococcus gordonii and Streptococcus sanguis. J Chemother 2000;12:379-84.

38. Hosgor-Limoncu M, Ermertcan S, Tasli H, et al. The post-antibiotic effects of linezolid against Gram-positive pathogens. Saudi Med J 2007;28:551-4.

39. Munckhof WJ, Giles C, Turnidge JD. Post-antibiotic growth suppression of linezolid against Gram-positive bacteria. J Antimicrob Chemother 2001;47:879-83.

40. Cattoir V, Leclercq R. Twenty-five years of shared life with vancomycin-resistant enterococci: is it time to divorce? J Antimicrob Chemother 2013;68:731-42.

41. Sastry S, Doi Y. Fosfomycin: Resurgence of an old companion. J Infect Chemother 2016;22:273-80.

42. Oh JT, Cassino C, Schuch R. Postantibiotic and SubMIC Effects of Exebacase (Lysin CF-301) Enhance Antimicrobial Activity against Staphylococcus aureus. Antimicrob Agents Chemother 2019;63:e02616-18.

(English Language Editor: L. Huleatt)

Cite this article as: Yan Y, Yang G, Li Y, Mao J, Wang S, Zhang N, Liu H, Huang X. Factorial design and post-antibiotic sub-MIC effects of linezolid combined with fosfomycin against vancomycin-resistant enterococci. Ann Transl Med 2022;10(3):148. doi: 10.21037/atm-21-4595 